\title{
Low-dose interferon- $\gamma$-producing human neuroblastoma cells show reduced proliferation and delayed tumorigenicity
}

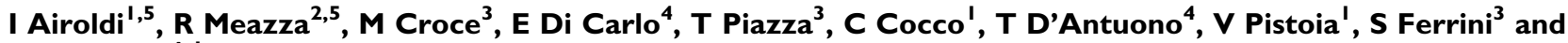 \\ MV Corrias*,1
}

'Laboratory of Oncology, Gaslini Institute, Largo Gaslini 5, 16148 Genoa, Italy; ${ }^{2}$ Laboratory of Clinical and Experimental Immunology, Gaslini Institute, Largo Gaslini 5, 16148 Genoa, Italy; ${ }^{2}$ Laboratory of Immunopharmacology, Istituto Nazionale per la Ricerca sul Cancro, Largo Benzi 12, 16132 Genoa, Italy; ${ }^{4}$ Department of Oncology and Neurosciences, G. D'Annunzio University, 66100 Chieti, Italy

Interferon- $\gamma$ (IFN- $\gamma$ ) directs T helper-I cell differentiation and mediates antitumour effects in preclinical models. However, high-dose IFN- $\gamma$ is toxic in vivo, and IFN- $\gamma$-transfected neuroblastoma (NB) cells secreting high amounts of the cytokine may be lost due to cell apoptosis or differentiation. Two human NB cell lines (ACN and SK-N-BE2(c)) differing as to genetic and phenotypic features were transfected with the human IFN- $\gamma$ gene and selected on the grounds of the low concentrations of IFN- $\gamma$ produced. In both IFN- $\gamma$ transfected cell lines, autocrine and paracrine activation of IFN- $\gamma$-mediated pathways occurred, leading to markedly reduced proliferation rate, to increased expression of surface HLA and CD40 molecules and of functional TNF binding sites. ACN/IFN- $\gamma$ cells showed a significantly delayed tumorigenicity in nude mice as compared to parental cells. ACN/IFN- $\gamma$ tumours were smaller, with extensive necrotic area as a result of a damaged and defective microvascular network. In addition, a significant reduction in the proliferation index was observed. This is the first demonstration that IFN- $\gamma$ inhibits in vivo proliferation of NB cell by acting on the tumour cell itself. This effect adds to the immunoregulatory and antiangiogenic activities operated by IFN- $\gamma$ in syngeneic tumourbearing hosts.

British Journal of Cancer (2004) 90, 2210-2218. doi:10.1038/sj.bjc.6601842 www.bjcancer.com

Published online 4 May 2004

(c) 2004 Cancer Research UK

Keywords: neuroblastoma; IFN- $\gamma$; transfection; cancer vaccine; tumour gene therapy

Neuroblastoma (NB), the most frequent extracranial solid tumour of childhood, arises from the neural crest, and the prognosis for patients presenting with disseminated disease at diagnosis is poor despite multimodal aggressive therapy (Brodeur et al, 1993).

Immunisation of cancer patients with cytokine-engineered tumour cells has been investigated at preclinical and clinical levels (Forni and Boggio, 1999; Pardoll, 2000; Parmiani et al, 2000; Wadhwa et al, 2002).

Several preclinical studies, performed in syngeneic mice with murine NB cell lines engineered to express different cytokines (Watanabe et al, 1989; Katsanis et al, 1994; Bausero et al, 1996), HLA antigens (Hock et al, 1995, 1996; Heuer et al, 1996; ) or costimulatory molecules (Katsanis et al, 1995; Heuer et al, 1996; Katsanis et al, 1996; Grossmann et al, 1997), have shown the therapeutic efficacy of this approach. In a xenogeneic model, we have previously shown that IL-2-transfected human NB cells inhibited the growth of parental tumour cells coinjected in nude mice (Corrias et al, 1998).

So far, three different phase I trials have been performed in NB patients unresponsive to conventional treatments, by employing

*Correspondence: Dr MV Corrias, Laboratorio Oncologia, Istituto G. Gaslini, L.go Gaslini, 5, 16/48 Genova, Italy;

E-mail: mariavaleriacorrias@ospedale-gaslini.ge.it

${ }^{5}$ These two authors contributed equally to the work

Revised I 8 December 2003; accepted 3 March 2004; published online 4 May 2004 either autologous or allogeneic NB tumour cells engineered to produce IL-2, alone or in combination with lymphotactin (Brenner et al, 2000; Rousseau et al, 2003; Bowman et al, 2003). In these trials, some of the vaccinated patients showed increase in leucocyte count, antibody levels and in NK activity.

Among the cytokines so far investigated, interferon (IFN)- $\gamma$ appears to be of particular interest due to its ability to upregulate surface expression of HLA class I molecules in human NB cells (Lampson et al, 1983; Ponzoni et al, 1993; Corrias et al, 2001) and to activate cytotoxic T-lymphocyte-mediated antitumour responses in a murine model (Watanabe et al, 1989).

However, studies in humans have shown that high-dose recombinant IFN- $\gamma$ administration is associated with serious side effects. Local low-dose release of cytokines, obtainable by gene transfer, may overcome systemic toxicity without impairing immune recognition of malignant cells by the immune system. In small cell lung carcinoma, a tumour that displays a downregulation of HLA class I antigen and shares neuroectodermal origin with $\mathrm{NB}$, IFN- $\gamma$ transfection have been demonstrated to enhance HLA class I surface expression and restore presentation of a MAGE-3 peptide to CTL (Traversari et al, 1997).

This approach appears feasible also for human NB cells since NB tumours express CTL-defined TAA, such as tyrosine hydroxylase ((Lode et al, 2000), MAGE-1 and 3 (Rimoldi et al, 1993; Corrias et al, 1996), NY-ESO (Soling et al, 1999; Rodolfo et al, 2003) and ALK (Lamant et al, 2000), and tumour-specific cytolytic $\mathrm{T}$ lymphocytes have been isolated from NB-bearing patients 
(Rosenthal et al, 1994; Bowman et al, 1998; Sarkar et al, 2001; Rousseau et al, 2003).

In addition to serving as an immunostimolatory molecule and exerting antiangiogenic effects through IP-10 production (Sgadari et al, 1996), IFN- $\gamma$ can sensitise tumour cells to apoptosis (Fulda and Debatin, 2002). Furthermore, it can induce expression of functional surface CD40 molecules on NB cells (Airoldi et al, 2003), making them susceptible to CD40L-mediated apoptosis.

In this study, we have established and characterised in vitro two low-dose IFN- $\gamma$-producing NB cell lines, which differ in their genetic and phenotypic features. Furthermore, we have tested tumorigenicity of $A C N / I F N-\gamma$ cell lines in a xenogeneic transplant model in nude mice and have addressed the mechanisms for delayed tumorigenicity.

\section{MATERIALS AND METHODS}

\section{Vectors and cell lines transfection}

The human IFN- $\gamma$ cDNA was amplified by RT - PCR starting from $1 \mu \mathrm{g}$ of total RNA extracted from PHA-activated peripheral blood lymphocytes. The sequences of PCR primers were: forward TGACAGGCTTAATTCTCTCGGAAACG, reverse TAGACTTAGGATCCAATATTGCAGGCAGGACAACC; a BamHI site is underlined. The cDNA was cloned in the XbaI blunted-BamHI sites of plasmid RSV.5 neo (kindly provided by Dr EO Long, NIH, Bethesda, MD, USA). Both ACN and SK-N-BE2(c) cell lines were transfected with $5 \mu \mathrm{g}$ of the RSV.5IFN- $\gamma$ (later refered as ACN/IFN$\gamma$ and $S K / I F N-\gamma$ ) or RSV.5 (later refered as $A C N /$ neo and $S K / n e o$ ) using cationic liposomes (DOTAP, Roche, Milano, Italy), according to instructions provided by the manufacturer. Stable transfectants were selected by growing the transfected cells in a medium containing $500 \mu \mathrm{g} \mathrm{ml}^{-1}$ of G-418 (Calbiochem, La Jolla, CA, USA).

\section{RNA extraction, RT - PCR analysis and RNAse protection assay (RPA)}

Total RNA was extracted using the RNeasy extraction kit (Qiagen, Hilden, Germany). In all, $1 \mu \mathrm{g}$ of total RNA, resuspended in sterile water, was retrotranscribed with oligo $d(T)$ by means of a commercial kit according to the procedure suggested by the manufacturer (Clontech, Palo Alto, CA, USA). At the end of the synthesis, the cDNA was diluted to $100 \mu \mathrm{l}$ and then $5 \mu \mathrm{l}$ was separately amplified with primers specific for IFN- $\gamma$, IP-10, IRF-1, NY-ESO, MAGE-1 and 3, TH, MYC-N, ALK and for the housekeeping gene G3PDH. The primers sequences, positions and accession number of the different sequences, size of the amplification products and annealing temperature are given in Table 1 . All the amplifications were performed for 35 cycles, after $10 \mathrm{~min}$ at $94^{\circ} \mathrm{C}$ to activate AmpliTaq gold polymerase (PerkinElmer Apllied Biosystem, Norwalk, CT, USA). Amplified products were analysed in a $2 \%$ agarose gel run in TBE buffer and stained with ethidium bromide. Specificity of the amplified products was determined by direct sequencing performed with the use of the Dye Terminator Cycle Sequencing Kit (ABI PRISM; Perkin-Elmer Applied Biosystem). Sequences were resolved and analysed on the ABI373A Sequence Apparatus (Perkin-Elmer Applied Biosystem).

RNAse protection assay was performed with $5 \mu \mathrm{glane}^{-1}$ total RNA and the Pharmingen probe hCR-3 kit (Pharmingen, San Diego, CA, USA), according to manufacturer's protocol. Products were resolved on $6 \%$ denaturing polyacrylamide gels and the protected fragments were visualised and quantitated using a PhosphorImager 445SI (Molecular Dynamics, Sunnyvale, CA, USA).

\section{Flow cytometry and cell cycle analysis}

Surface expression of HLA molecules was analysed by indirect immunofluorescence and cytofluorimetric analysis using the W6.32 mAb (anti HLA I, kindly provided by Dr S Ferrone, Roswell Park Cancer Center, Buffalo NY, USA) and the D1.12 mAb (anti HLA II, kindly provided by Dr R Accolla, University of Insubria, Italy). An FITC-conjugated goat anti mouse IgG2a was used as second-step reagent. Surface expression of CD40 was analysed by means of PE-conjugate anti CD40 mAb from Diaclone SA, Besançon, France. Controls for anti HLA I and HLA II were purified murine IgG2a (Southern Biotechnologies Associates, Birmingham, AL, USA) of irrelevant specificity. Control for antiCD40 was PE-conjugated, isotype-matched $\mathrm{mAb}$ of irrelevant specificity. Intracellular staining for anti Ki-67 was performed by incubating cells in PBS containing $0.1 \%$ saponin and anti $\mathrm{Ki}-67$ antibody (clone MIB-1, Dako, Glostrup, Denmark) or the corresponding control isotype (purified murine IgG1 of irrelevant specificity from Southern Biotechnologies Associates). An FITCconjugated goat anti mouse IgG1 was used as second-step reagent. Samples were analysed with the FACScan (BD BiosciencesMountain View, CA, USA). Cells were scored using a FACScan analyzer (Becton-Dickinson, San Jose, CA, USA) and data were processed using CellQuest software (Becton-Dickinson). The threshold line was based on the maximum staining obtained with irrelevant isotype-matched $\mathrm{mAb}$, used at the same concentration as test mAb. Negative cells were defined such that less than $1 \%$ of cells stained positive with control mAbs. Cells labeled with test antibody that were brighter than those stained with isotypic control antibody were defined as positive.

Cell cycle analysis was performed by incubating $10^{5}$ cells in PBS containing $40 \mu \mathrm{g} \mathrm{ml}^{-1}$ propidium iodide, $0.1 \%$ Triton X-100 and $400 \mu \mathrm{g} \mathrm{ml}^{-1}$ RNase A for $10 \mathrm{~min}$ at $37^{\circ} \mathrm{C}$. Cells were analysed by flow cytometry using CellQuest software, after 10 additional minutes at room temperature.

\section{Cytokine production}

Supernatants of semiconfluent, G418-resistant, IFN- $\gamma$-transfected NB cells were collected after $48 \mathrm{~h}$ and analysed for human IFN- $\gamma$

Table I Sequence of primers used in RT-PCR analysis and size of the amplification products

\begin{tabular}{|c|c|c|c|c|c|c|c|}
\hline Gene & Accession no. & Forward & Position & Reverse & Position & Temp & Bp \\
\hline $\mathrm{IFN}-\gamma$ & $\times 13274$ & 5'ATTGAATTCACGATGAAATATACAAGTTA & $106-125$ & 5'-ATTCTCGAGCAGGCAGGACAACCATTACT & $605-624$ & $60^{\circ} \mathrm{C}$ & 518 \\
\hline IP- 10 & $\times 02530$ & 5'-GGAACCTCCAGTCTCAGCACC & $46-66$ & 5'-GGATGATGAACATTAACCTTCC & $536-559$ & $58^{\circ} \mathrm{C}$ & 513 \\
\hline |RF- & NM_002198 & 5'-GCTCCACTCTGCСTGATGACC & $658-678$ & 5'-GAGGAATAAGAGGGGCCCAGG & $1183-1203$ & $59^{\circ} \mathrm{C}$ & 545 \\
\hline $\mathrm{TH}$ & NM_0000360 & 5'TGTCAGAGCTGGACAAGTGT & $528-547$ & 5'-GATATTGTCTTCCCGGTAGC & $826-807$ & $60^{\circ} \mathrm{C}$ & 299 \\
\hline MAGEI & AYI 48486 & 5'-CGGCCGAAGGAACCTGACCCAG & $1-22$ & 5'-GCTGGAACCCTCACTGGGTTGCC & $399-421$ & $60^{\circ} \mathrm{C}$ & 420 \\
\hline MAGE3 & HSU03735 & 5'-TGGAGGACCAGAGGCCCCC & $2276-2294$ & 5'-GGACGATTATCAGGAGGCCTGC & $3059-3080$ & $60^{\circ} \mathrm{C}$ & 804 \\
\hline NY-ESO & NM_00I 327 & 5'GCCGCCTGCTTGAGTTCTAC & $342-361$ & 5'-CTCCTCCAGCGACAAACAAT & $718-737$ & $60^{\circ} \mathrm{C}$ & 395 \\
\hline MYC-N & NM_005378 & 5'TGTCGGTTGCAGTGTTGGAGG & $22-42$ & 5'-TGTCGGTTGCAGTGTTGGAGG & $247-267$ & $60^{\circ} \mathrm{C}$ & 245 \\
\hline ALK & XM_055726 & 5'-GCTGAGCAAGCTCCGCACCTCGAC & $4 \mid 44-4167$ & 5'-CCCGCCATGAGCTCCAGCAGGATG & $4486-4509$ & $60^{\circ} \mathrm{C}$ & 365 \\
\hline GAPDH & NM_002046 & 5'-ACATCGCTCAGAACACCTATGG & $16-37$ & 5'-GGGTCTACATGGCAACTGTGAG & $119 \mid-1212$ & $60^{\circ} \mathrm{C}$ & 1196 \\
\hline
\end{tabular}


production by a commercially available ELISA kit (R\&D System, Minneapolis, MN, USA). Cells were counted and results are referred to $48 \mathrm{~h}$ production from $10^{6}$ cells.

\section{TNF- $\alpha$ receptor binding assay}

Cells were seeded in triplicate at a density of $10^{6}$ well $^{-1}$ in $2 \mathrm{ml}$ medium in six-well plates. After $24 \mathrm{~h}$, cells were washed twice and then incubated for $2 \mathrm{~h}$ at $4{ }^{\circ} \mathrm{C}$ in cold medium containing $80-$ $120 \mathrm{pm}^{125}$ I-labeled hrTNF- $\alpha$ (Sorin Pharmaceuticals, Verona, Italy) in the presence or absence of increasing concentrations of unlabelled hrTNF- $\alpha$ (a generous gift of Boehringer Mannheim, Mannheim, Germany). Afterwards, cells were washed twice with ice-cold medium, detached with ice-cold PBS and washed twice by centrifugation. The pellet-associated ${ }^{125} \mathrm{I}$ was counted in a $\gamma$ counter (LKB, Uppsala, Sweden) with an estimated efficiency of $80 \%$. Cell number was determined by counting cells in a hemocytometer. To evaluate the binding affinity and number of site per cell, the ligand displacement curves were subjected to Scatchard analysis by means of the Ligand program by considering TNF- $\alpha$ as a trimer, as previously described (Montaldo et al, 1994).

\section{Coculture of parental and IFN- $\gamma$-transfected cells}

Parental cells $\left(5 \times 10^{5}\right)$ were seeded on the bottom plate and $5 \times 10^{5}$ of either the empty vector- or the IFN- $\gamma$-transfected cells were seeded on the upper plate of a transwell (Costar, Cambridge, MA, USA). The two chambers were separated by a policarbonate filter with pore size of $0.4 \mu \mathrm{m}$. Afterdays of coculture, the upper well was discarded and the parental cells on the bottom plate were detached and analysed by flow cytometry or by RT - PCR analysis.

\section{Nude mice studies}

Pathogen-free female athymic (nu/nu) mice, 6-8 weeks old, were obtained from Harlan Italy (San Pietro al Natisone, Italy). Animal experiments, performed according to the National Regulation on Animal Research Resources, were approved by the Review Board of the Istituto Nazionale per la Ricerca sul Cancro. Mice were housed under sterile conditions and received autoclaved food and water. Animals (seven for each group) were injected subcutaneously with $2 \times 10^{7}$ parental ACN or transfected $A C N /$ neo or $A C N / I F N-\gamma$ cells. Tumour size, measured twice a week with a caliper, was expressed as a multiple of the wider and smaller diameters. Statistical analysis was performed by the MannWithney test.

\section{Morphological analysis}

$A C N /$ neo and $A C N / I F N-\gamma$ tumours were removed at day 14 post injection (p.i.), fixed in 10\% neutral-buffered formalin, embedded in paraffin, sectioned at $4 \mu \mathrm{m}$, and stained with haematoxylineosin for histological evaluation. For immunohistochemistry, formalin-fixed, paraffin-embedded sections were incubated with anti-human Ki-67 (clone MIB-1, Dako) or anti laminin (Ab No. 078P, BioGenex, San Ramon, CA, USA) Abs. The rates of proliferating (immunoreactivity for Ki-67) cells were obtained by counting the number of positive cellsper number of total cells in the viable neoplastic tissue excluding areas of tissue necrosis under a microscope at $\times 400$ in a $0.180 \mathrm{~mm}^{2}$ field. Differences in the number of Ki-67-positive cells were evaluated by Student's $t$-test.

\section{RESULTS}

\section{Stable IFN- $\gamma$ transfection of NB cell lines}

Several NB cell lines were transfected with the recombinant RSVneo/human IFN- $\gamma$ gene or the empty vector. All the

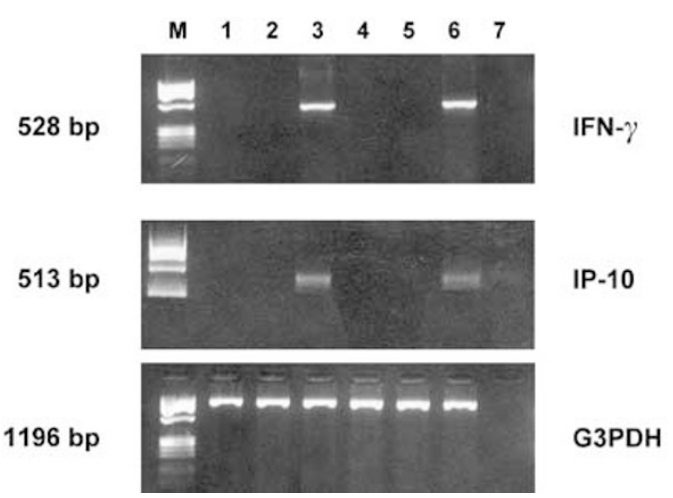

Figure I IFN- $\gamma$ and IP-IO gene expression in parental and IFN- $\gamma$ transfected NB cell lines. RT-PCR analysis performed on parental, empty vector- and IFN-transfected SK-N-BE2(c) (lanes I-3) and ACN (lanes 46) cells, and water as negative control (lane 7). Size of the expected fragments is indicated on the left. G3PDH amplification products are shown in the lower panel. $M=\Phi \chi \mid 74$ Hae/digest molecular weigh marker.
A

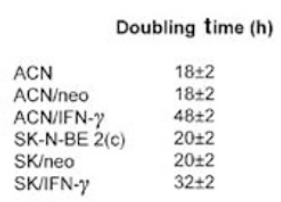

(1)

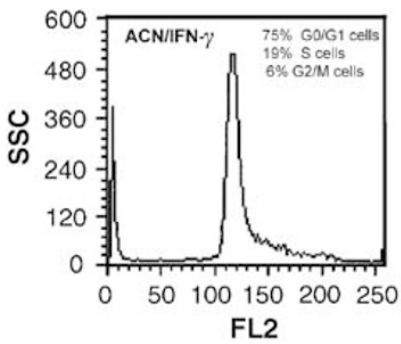

D

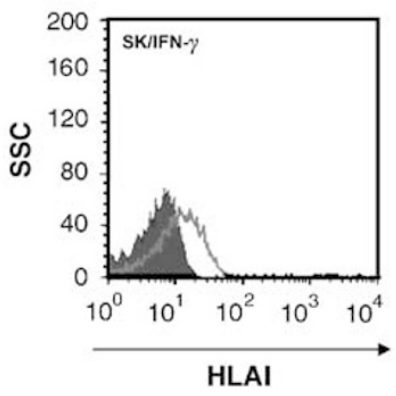

B

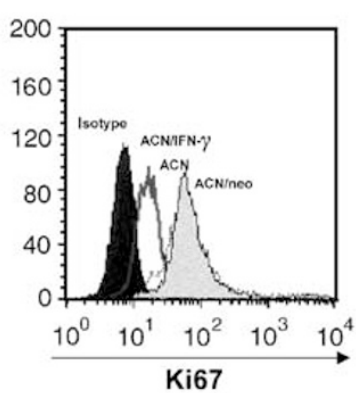

C

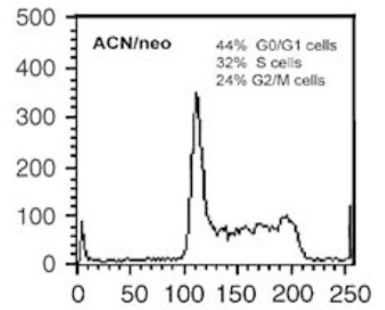

E

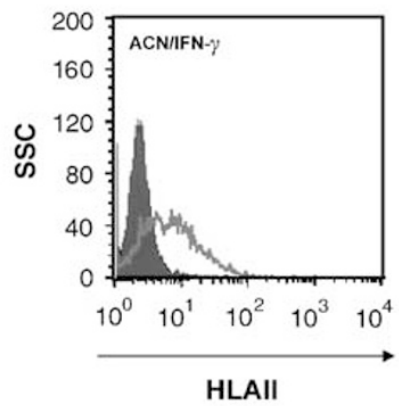

Figure 2 In vitro effects of IFN- $\gamma$ transfection on NB cells. (A) Doubling time of IFN- $\gamma$-transfected, empty vector-transfected and parental NB cells, assessed by counting cells every day for a week (B) Proliferation index of ACN, ACN/IFN- $\gamma$ and ACN/neo, assessed by Ki-67 staining. (C) Cell cycle analysis of ACN/IFN- $\gamma$ (left panel) and ACN/neo (right panel) cells. (D) HLA class I surface expression of SKIIFN- $\gamma$ cells (dark profile: SK/neo; open profile: SKIIFN- $\gamma$ ). (E) HLA class II surface expression of ACN/IFN- $\gamma$ cells (dark profile: $A C N /$ neo; open profile: $A(N / I F N-\gamma)$, as assessed by flow cytometry. 
Table 2 Growth rate, TNF receptor surface expression and effect of TNF- $\alpha$ treatment on growth rate of parental, vector- and IFN-transfected NB cells

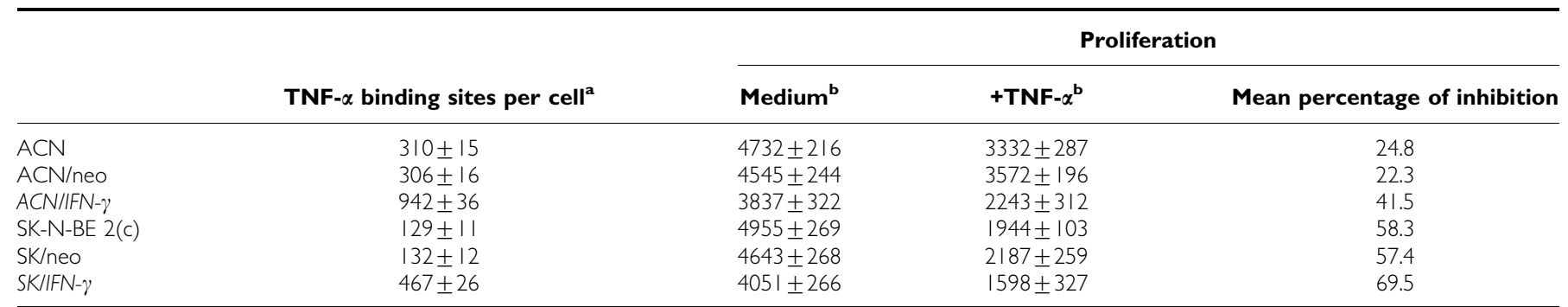

a see Materials and Methods for details on Scatchard analysis. $\left.{ }^{b} \mathrm{I} \mathrm{h}{ }^{3} \mathrm{H}\right]$ thymidine incorporation measured in $5 \times 10^{4}$ cells cultured for 2 days in medium or in medium containing hrTNF- $\alpha$ at $100 \mathrm{IU} \mathrm{ml}^{-1}$. All the results are the mean \pm s.d. of three independent experiments, each carried out in duplicate.

transfectants producing IFN- $\gamma$ concentrations in culture super-

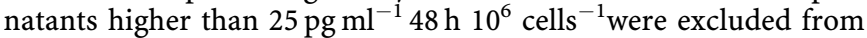
the study. Two stable, low-dose IFN- $\gamma$-producing transfected cell lines, ACN and SK-N-BE2(c), were established. ACN cells lack MYC-N amplification and 1p3.6 deletion (Thiele, 1999), but express MAGE-1 and -3 (Corrias et al, 1996), as well as NY-ESO and ALK (this paper, see below). In addition, ACN cells are tumorigenic in nude mice (Corrias et al, 1998). On the contrary, SK-N-BE2(c) cells are MYC-N amplified and 1p deleted (Thiele, 1999), express MYC-N and TH (this paper, see below) and are not tumorigenic in nude mice.

Both stable IFN- $\gamma$ transfectants expressed human IFN- $\gamma$-specific transcripts when tested by RT - PCR (Figure 1). IFN- $\gamma$ production in culture supernatants from IFN- $\gamma$-transfected ACN and SK-N$\mathrm{BE} 2$ (c) cells was 19.2 and $18.5 \mathrm{pg} \mathrm{ml}^{-1} 48 \mathrm{~h}^{-1} 10^{6} \mathrm{cells}^{-1}$, respectively, as assessed by ELISA.

\section{In vitro characterisation of IFN- $\gamma$ transfectants}

IFN- $\gamma$-transfected cells from both the cell lines displayed a more differentiated phenotype, that is presence of neurites, than their parental counterparts (data not shown), as observed following treatment with hr IFN- $\gamma$ (Ponzoni et al, 1992).

IFN- $\gamma$ transfection activated IFN-regulated pathways in both NB cell lines, as indicated by de novo expression of the IFN-inducible IP-10 gene transcript (Figure 1). Moreover, both $A C N / I F N-\gamma$ and $S K / I F N-\gamma$ transfectants displayed a dramatic increase in their doubling time, as evaluated by cell count (Figure 2A). In order to understand the mechanism(s) underlying cell growth inhibition, we analysed the effect of released IFN- $\gamma$ on proliferation rate and apoptosis. IFN $-\gamma$ transfection significantly $(P<0.005)$ reduced the proliferation rate, assessed by Ki-67 staining, of $A C N / I F N-\gamma$ cells, as compared to parental and vector-transfected cells (Figure $2 \mathrm{~B}$ ). Indeed, cell cycle analysis showed that $A C N / I F N-\gamma$ cells were mostly arrested in G0/G1 phase (Figure 2C), as compared to vector-transfected $A C N / n e o$ cells. On the contrary, IFN- $\gamma$ transfection did not significantly modify the amount of apoptotic cells in culture (data not shown). Similar results were obtained with the SK-N-BE2(c) transfectants (data not shown).

IFN- $\gamma$ transfection induced, in both cell lines, remarkable changes in surface expression of HLA molecules. In the SK-N-BE2(c) cell line, which constitutively expresses very low amounts of HLA class I and no HLA class II molecules (Corrias et al, 2001), IFN- $\gamma$ transfection produced a clear-cut increase in HLA class I antigen (Figure 2D) and no changes in HLA class II expression (not shown) with respect to $S K / n e o$ cells. In the ACN cell line, which constitutively expresses HLA class I and low amounts of HLA class II molecules (Corrias et al, 2001), increased expression of HLA class II antigens was detected (Figure 2E) with respect to the ACN/neo cells.

Consistent changes also occurred in the surface expression of TNF- $\alpha$ binding sites, as assessed by Scatchard analysis using radiolabelled hrTNF- $\alpha$ (Ponzoni et al, 1992; Montaldo et al, 1994).
A

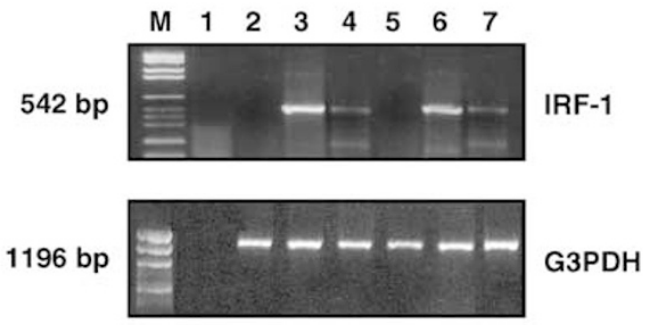

B

C

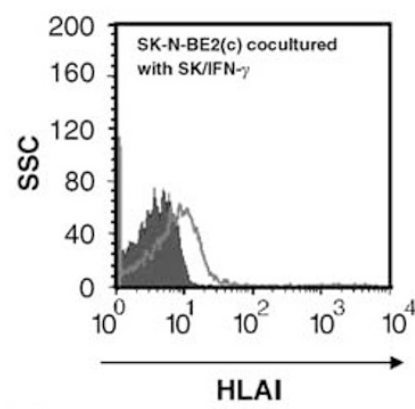

D
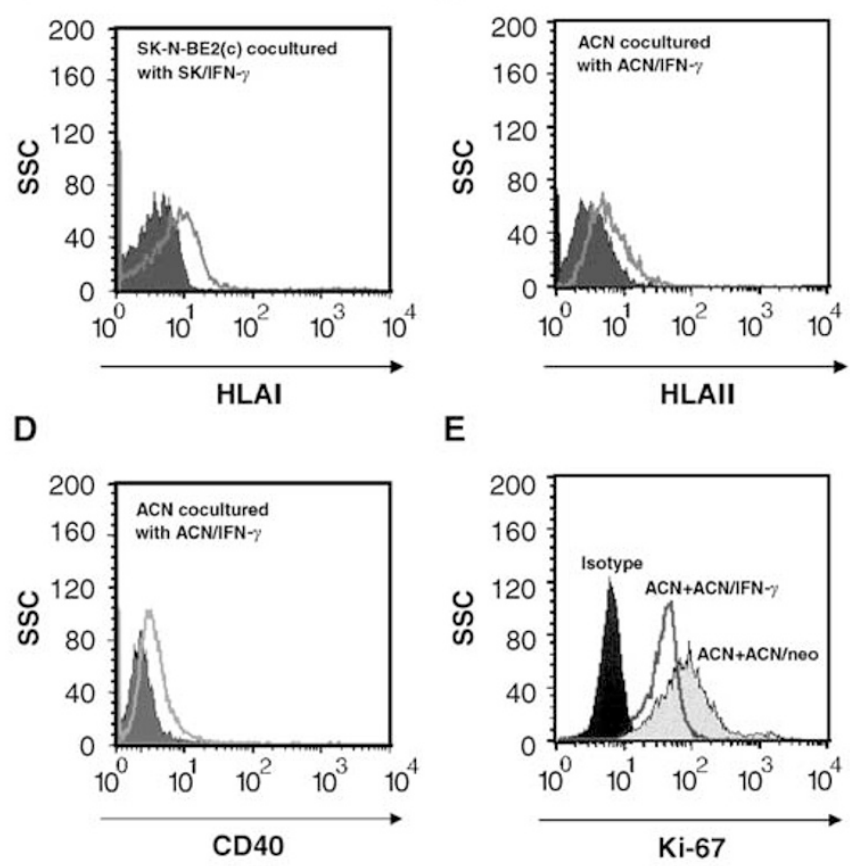

Figure 3 Effects of IFN- $\gamma$ transfection on cocultured parental NB cells. (A) RT-PCR analysis of IRF-I gene expression. $M=$ molecular weigh markers. Negative control (lane I), ACN cells (lane 2), ACN/IFN- $\gamma$ cells (lane 3), ACN cells cocultured with ACN/IFN- $\gamma$ cells (lane 4), SK-N-BE2(c) cells (lane 5), SK/IFN- $\gamma$ cells (lane 6), SK-N-BE2(c) cocultured with SKIIFN- $\gamma$ cells (lane 7). G3PDH gene expression is shown below. Size of the expected fragment is indicated on the left side. (B) HLA class I surface expression of parental SK-N-BE2(c) cells cocultured with SK/IFN- $\gamma$ cells for $72 \mathrm{~h}$ (dark profile: irrelevant murine IgG2a; open profile anti HLA I W6.32 mAb). (C) HLA class II surface expression of parental ACN cells cocultured with ACN/IFN- $\gamma$ cells for $72 \mathrm{~h}$ (dark profile: irrelevant murine IgG2a; open profile: anti HLA || DI. 12 mAb). (D) CD40 surface expression of parental ACN cells cocultured with ACN/IFN- $\gamma$ cells for $72 \mathrm{~h}$ (dark profile: $\mathrm{PE}$-conjugated $\mathrm{mAb}$ of irrelevant specificity; open profile: $\mathrm{PE}$-conjugated anti CD40 mAb). (E) Ki-67 staining of ACN cells cocultured with ACN/IFN$\gamma$ (open profile) or ACN/neo (grey profile), as assessed by flow cytometry. Dark profile: ACN cells stained with an isotype-matched irrelevant mAb. 


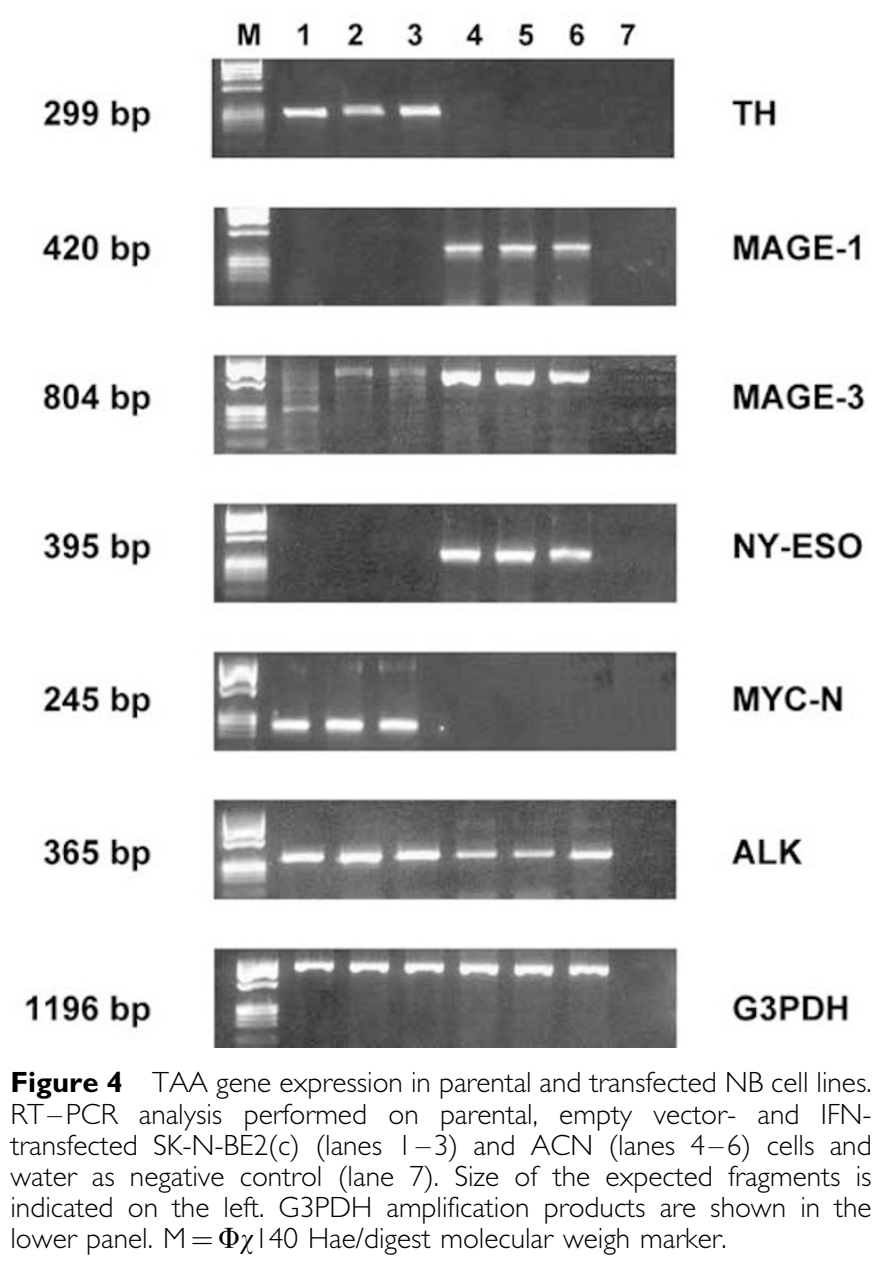

As shown in Table 2, IFN- $\gamma$-transfected cells expressed three times more binding sites than parental and empty vector-transfected cells. Furthermore, when the IFN- $\gamma$ transfectants were treated with hrTNF- $\alpha$, their growth rate was further reduced (Table 2). Inhibition of cell proliferation was higher than that observed in the parental or empty vector-transfected cells (Table 2), although it was more evident for $A C N / I F N-\gamma$ cells than for $S K / I F N-\gamma$ cells. These findings confirmed the occurrence of a synergistic effect between IFN- $\gamma$ and TNF- $\alpha$ at inducing differentiation of NB cells (Ponzoni et al, 1992; Montaldo et al, 1994).

\section{Effect of IFN- $\gamma$ released by IFN- $\gamma$ transfectants on cocultured parental cells}

To test whether the low amounts of IFN- $\gamma$ released by IFN- $\gamma$ transfectants was sufficient to upregulate, by a paracrine mechanism, IFN- $\gamma$-inducible gene expression in the parental cells, coculture in a transwell system was performed.

Both parental cell lines showed induction of IRF-1 gene expression, as assessed by RT-PCR analysis, upon coculture for 3 days with the corresponding IFN- $\gamma$ transfectants (Figure 3A).

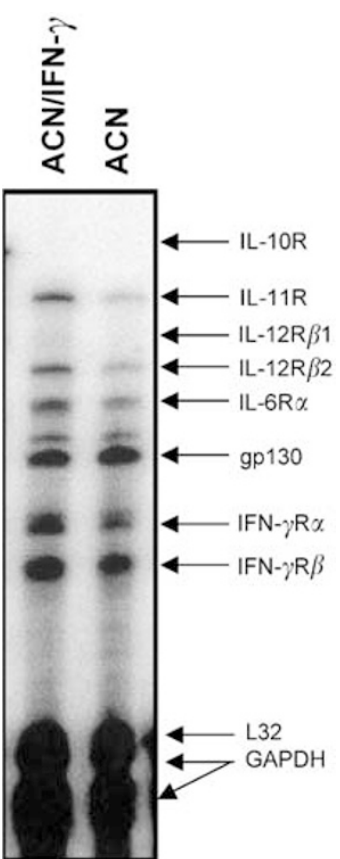

Figure 5 Cytokine receptor gene expression in parental and IFN $\gamma$ transfected ACN cells as assessed by RPA. RNase protection assay was performed with $5 \mu \mathrm{g}$ lane ${ }^{-1}$ total RNA and the Pharmingen probe hCR-3 kit.

IRF-1 is the transcription factor necessary and sufficient to enhance expression of all the IFN- $\gamma$-inducible genes (Loh et al, 1992). Following coculture with paired IFN- $\gamma$ transfectants, parental SK-N-BE2(c) cells expressed HLA class I molecules (Figure 3B) and parental ACN cells HLA class II molecules (Figure 3C). No modification in HLA surface expression was observed when the parental ACN and SK-N-BE2(c) cells were cocultured with the empty vector-transfected cells (not shown).

We have recently demonstrated that treatment of several NB cell lines with hrIFN- $\gamma$ induced surface expression of CD40 (Airoldi et al, 2003). ACN/IFN- $\gamma$ also showed enhanced CD40 gene and surface expression (Airoldi et al, 2003). ACN/IFN- $\gamma$ cells cocultured with parental ACN cells induced CD40 surface expression on these latter cells (Figure 3D), further confirming that a paracrine effect was produced by the low amount of IFN- $\gamma$ released by the NB transfectants. Similar results were obtained when ACN cells were cocultured with $S K / I F N-\gamma$ cells and, conversely, the latter cells were cocultured with $A C N / I F N-\gamma$ cells (data not shown).

Finally, IFN- $\gamma$ released by $A C N / I F N-\gamma$ transfectants significantly reduced $(P<0.005)$ the proliferation rate of cocultured parental ACN cells, as assessed by Ki-67 staining (Figure $3 \mathrm{E}$ ). No change in the percentage of apoptotic cells was observed under this conditions (data not shown). Similar results were obtained in parental SK-NBE2(c) cells cocultured with SK/IFN- $\gamma$ (data not shown).

\section{Expression of TAA genes in IFN- $\gamma$-transfected NB cells}

Next, we investigated the effects of IFN- $\gamma$ transfection on the expression of different TAA genes, as assessed by RT-PCR. As

Figure 6 Growth rate, histological and immunohistochemical features of parental, ACN/neo and ACN/IFN- $\gamma$ cells in nude mice. (A) Growth rate in vivo. $2 \times 10^{7}$ parental $(O-O)$, vector-transfected $(\square-\square)$ or IFN-transfected ACN $(\diamond-\diamond)$ cells were inoculated subcutaneously in the left flank of nude mice. Tumour growth was measured twice a week with a caliper. Results are the mean of two independent experiments, each one performed with five animals per group. (B) Photograph of ACN/neo and ACN/IFN- $\gamma$ tumours taken 14 days post injection (p.i.). (C) Histological and immunohistochemical analysis of ACN/neo and ACN/IFN- $\gamma$ tumours developed I 4 days p.i. H\&E: ACN/neo and ACN/IFN- $\gamma$ tumour sections stained with haematoxylin-eosin. Small nests of round to oval cells with abundant amphophilic cytoplasm and nucleolated nuclei are shown. ACN/IFN- $\gamma$ tumours showed extensive areas of necrosis ( $\mathrm{N}$ ) frequently infiltrated by reactive cells (arrows). Ki-67: Tumour sections stained with anti Ki-67 mAb. Laminin: Tumour sections stained with anti-laminin mAb. Magnification is $\times 400$. 
shown in Figure 4, SK/IFN- $\gamma$ cells contained the transcripts of MYC-N, ALK and TH genes as parental SK and SK/neo cells, while $A C N / I F N-\gamma$ cells displayed expression of ALK, NY-ESO, MAGE-1 and -3 mRNAs as parental ACN and ACN/neo cells (Figure 4).

\section{Expression of cytokine receptor genes in IFN- $\gamma$-transfected} NB cells

We next tested by RNAse protection assay whether IFN- $\gamma$ transfection modified gene expression of different cytokine

A

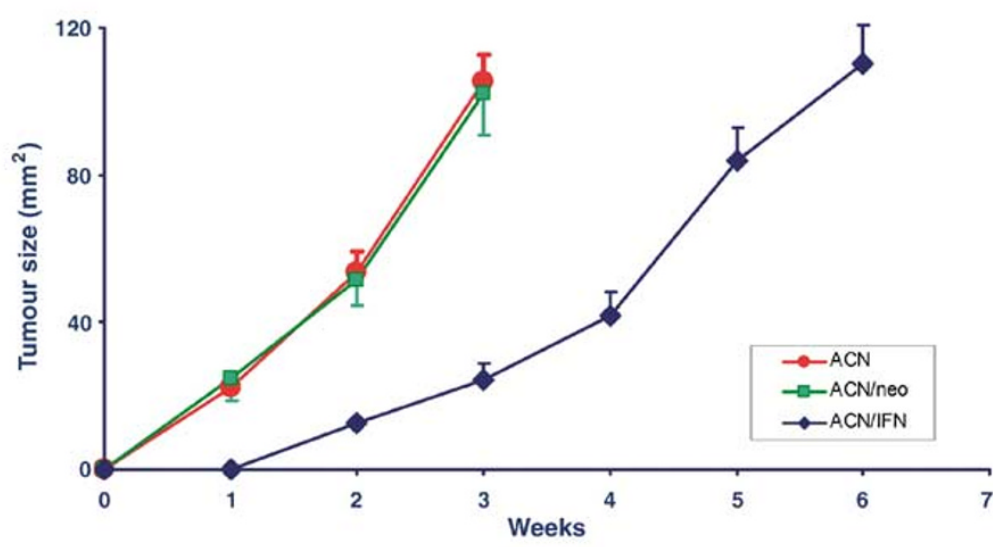

B

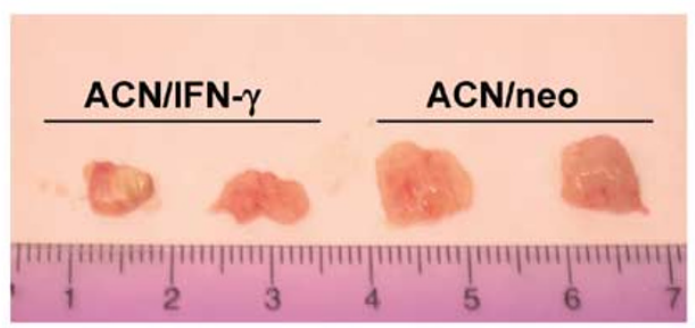

C

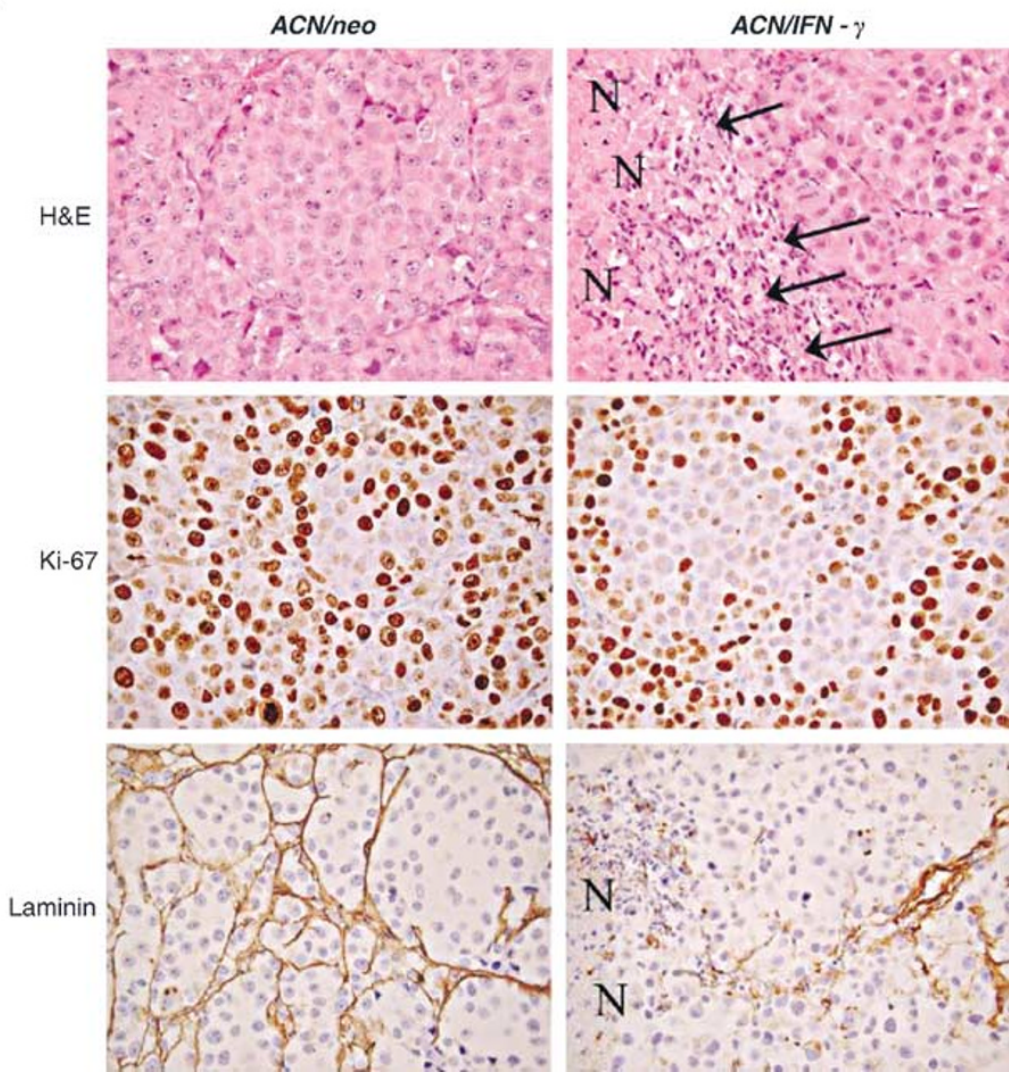


receptors, including IFN- $\gamma$ itself. As shown in Figure 5, ACN/ $I F N-\gamma$ cells showed enhanced mRNA expression of IFN- $\gamma$ receptor (R) $\alpha$, IL-6R $\alpha$, IL-11R and IL-12R $\beta 2$. Notably, ACN and SK-N-BE2(c) cells did not express IL-12R $\beta 1$, irrespective of IFN- $\gamma$ gene transfection (Figure 5). In addition, expression of two proinflammatory cytokines, IL- 8 and TNF- $\alpha$, known to be expressed by NB cell lines (Nitta et al, 1994; Yang et al, 1994), and of two anti-inflammatory cytokines, IL-10 and TGF- $\beta 1$, known to inhibit antitumour responses (Hsieh et al, 2000) was not modified by IFN- $\gamma$ gene transfection (data not shown).

\section{Tumorigenicity of ACN/IFN- $\gamma$ in nude mice and histopathological features of tumours}

Species specificity of IFN- $\gamma$ and IP-10 does not allow one to test elicitation of antitumour responses in immunodeficient mice bearing human IFN- $\gamma$-transfected cells. Therefore, we tested whether IFN- $\gamma$ transfection affected the tumorigenicity of ACN cell line by acting through autocrine and paracrine mechanisms at the level of the tumour cell themselves. Parental, ACN/neo and $A C N / I F N-\gamma$ cells were injected subcutaneously in nude mice and tumour growth was measured. The in vivo growth of $A C N / I F N-\gamma$ cells was significantly $(P<0.005)$ slower than that of parental or vector-transfected ACN cells in vivo (Figure 6A). To address whether this was a consequence of reduced proliferation rate, histopatological analyses were performed on tumours removed at days 14 post injection, when macroscopic differences in the volume of the $A C N / I F N-\gamma$ and $A C N / n e o$ tumours were observed (Figure 6B).

The tumour masses developed 14 days after s.c. injection of $A C N /$ neo cells into nu/nu mice were formed by small nests of closely packed round or oval cells with abundant amphophilic cytoplasm and vesciculated nuclei containing one or more conspicuous nucleoli (Figure 6C, H\&E). The pattern of growth was vaguely nodular as a result of the presence of delicate, incomplete fibrous septa. Small necrotic foci were a rather constant feature. However, mitotic figures were also frequent (Figure 6C, H\&E).

Mice injected with $A C N / I F N-\gamma$ cells developed tumours, which were smaller in size (Figure 6B) and showed extensive necrotic areas (Figure 6C, H\&E, N) often infiltrated with granulocytes and macrophages phagocyting cell debris (Figure 6C, H\&E arrows). The proliferation index, assessed by Ki-67 immunoreactivity in the viable neoplastic tissue excluding areas of tissue necrosis, was significantly lower $(P<0.005)$ than in $A C N /$ neo tumours $(66.5 \pm 4.0$ vs $80.2 \pm 5.4 \%$ ) (Figure $6 \mathrm{C}$, Ki-67).

In addition, staining for laminin, a marker of the basement membrane, revealed that the vascular architecture was mostly intact in ACN/neo tumours, while aspects of focal basement membrane destruction and alterations in the microvascular architecture were frequent in the inner portion of $A C N / I F N-\gamma$ tumour (Figure 6C, Laminin).

\section{DISCUSSION}

In this study, two human NB cell lines differing in their genetic and phenotypic features were transfected with the human IFN- $\gamma$ gene. These transfectants, which produced low amounts of the cytokine in culture supernatants, displayed dramatic reduction in their proliferation rate, increased expression of functional TNF- $\alpha$ binding sites, HLA and CD40 surface molecules, similarly to that observed in vitro following treatment of $\mathrm{NB}$ cell lines with hrIFN- $\gamma$ (Ponzoni et al, 1992; Montaldo et al, 1994; Airoldi et al, 2003).

More interestingly, all these changes occurred, by a paracrine mechanism, in parental cells cocultured with the
IFN- $\gamma$ transfectants. In addition, IFN- $\gamma$-transfected ACN cells showed a significantly reduced tumorigenicity, as compared to parental and empty vector-transfected cells. This result was obtained in a model, that is, human tumour cell xenograft in nude mice, which precludes any effect of the transfected cytokine on the mouse immune system, due to the species specificity of human IFN- $\gamma$. Thus, the slow proliferation rate of NB cells observed in vivo was attributable to autocrine and paracrine effects of the transfected cytokine on the tumour cells themselves.

Since human IFN- $\gamma$ is species specific, the antiangiogenic effect we observed likely resulted from anti-angiogenic mediators produced by the tumour cells. The defective intratumoral vascular network may lead to extensive necrosis followed by reactive influx of phagocytosing cells. These results indicated that IFN- $\gamma$ transfection of human NB cells may achieve not only activation of immune antitumour responses, as already demonstrated by others, but also affect NB tumour growth per se, by inhibiting $\mathrm{NB}$ proliferation and exploiting an antiangiogenic effect.

Murine and human IFN- $\gamma$ gene-engineered NB cells have already been produced (Watanabe et al, 1989; Coze et al, 1995; Katsanis et al, 1995; Bausero et al, 1996). Murine IFN- $\gamma$ transfected NB cells were shown to induce specific CTL effectors in tumour-bearing syngeneic mice (Watanabe et al, 1989). So far, human IFN- $\gamma$-transduced NB cells have been characterised in vitro for phenotypic features (Coze et al, 1995; Ucar et al, 1995) and ability to stimulate alloreactive T-cell responses in mixed lymphocyte/tumour cell cultures (Coze et al, 1995; Coll et al, 1997).

The strategy of selecting low producing IFN- $\gamma$-transfected NB cells for this study was followed to prevent loss of transfectants, as already described by others (Coze et al, 1995; Ucar et al, 1995), due to the potent induction of NB tumour cell differentiation (Ponzoni et al, 1992; Ponzoni et al, 1993; Montaldo et al, 1994) and sensitisation to apoptosis (Fulda et al, 2001; Banelli et al, 2002; Fulda and Debatin, 2002) caused by high level secretion of the cytokine. The IFN- $\gamma$-transfected NB cell lines described here have been maintained in culture for prolonged period of time without substantial changes in their characteristics.

Minimal amounts of IFN- $\gamma$ were indeed sufficient to induce remarkable changes in surface expression of several molecules, such as HLA, TNF-R and CD40, in cocultured parental NB cells throughout paracrine mechanism. In this respect, we have recently shown that incubation of CD40-positive NB cells with soluble or insoluble CD40L leads to tumour cell apoptosis (Airoldi et al, 2003). These observations may be relevant in the perspective of the design of a vaccination protocol for patients using allogeneic cell lines transfected with the human IFN- $\gamma$ gene. Thus, lowdose IFN- $\gamma$ secreted by the latter cells locally injected at the tumour resection site (where a few tumour cells may escape surgical intervention) may promote presentation of peptide-derived tumour-associated antigen to $\mathrm{T}$ lymphocytes leading to specific anti NB responses. In addition, CD40L-induced apoptosis of tumour cells may contribute to their elimination, provided that CD40L-positive activated $\mathrm{T}$ lymphocytes are recruited to the tumour site.

Furthermore, low secretion of IFN- $\gamma$ may help limit in vivo side effects elicited by high doses of the cytokine, such as systemic toxicity and altered T-lymphocyte polarisation and/or proliferation, as recently reported by Refaeli et al (2002).

Another issue here investigated is the expression of different cytokine receptors in IFN- $\gamma$-transfected cells. Notably, IL-11R and IL-6R transcripts were clearly upregulated in transfected cells; both IL-6 and IL-11 belong to the gp-130-associated family of neuroactive cytokines. Furthermore, upregulation of the transcripts for both chains of the IFN $-\gamma \mathrm{R}$ was observed in transfected cells, in accordance with previous studies. These results indicate 
that introduction of exogenous IFN- $\gamma$ gene in NB cells may modify their sensitivity to other cytokines, possibly present in the tumour microenvironment.

In conclusion, IFN- $\gamma$-transfected allogeneic NB cell lines may be envisaged as a delivery system of the cytokine to residual tumour cells in a clinical setting. Low-dose IFN- $\gamma$ secretion would limit its systemic toxicity while retaining most immunomodulatory and antiangiogenic activities.

\section{REFERENCES}

Airoldi I, Lualdi S, Bruno S, Raffaghello L, Occhino M, Gambini C, Pistoia V, Corrias MV (2003) Expression of costimulatory molecules in human neuroblastoma. Evidence that CD40+ neuroblastoma cells undergo apoptosis following interaction with CDL40L. Br J Cancer 88: $1527-1536$

Banelli B, Casciano I, Croce M, Di Vinci A, Gelvi I, Pagnan G, Brignole C, Allemanni G, Ferrini S, Ponzoni M, Romani M (2002) Expression and methylation of CASP8 in neuroblastoma: identification of a promoter region. Nat Med 8: 1333-1335 author reply 1335

Bausero MA, Panoskaltsis-Mortari A, Blazar BR, Katsanis E (1996) Effective immunization against neuroblastoma using double-transduced tumor cells secreting GM-CSF and interferon-gamma. J Immunother Emphasis Tumor Immunol 19: 113-124

Bowman L, Grossmann M, Rill D, Brown M, Zhong WY, Alexander B, Leimig T, Coustan-Smith E, Campana D, Jenkins J, Woods D, Kitchingman G, Vanin E, Brenner M. (1998) IL-2 adenovector-transduced autologous tumor cells induce antitumor immune responses in patients with neuroblastoma. Blood 92: 1941-1949

Bowman LC, Brenner MK, Strother D (2003) Local and systemic effects of an allogeneic tumor cell vaccine combining transgenic human lymphotactin with interleukin-2 in patients with advanced or refractory neuroblastoma. Blood 101: 1718-1726

Brenner MK, Heslop H, Krance R, Horowitz M, Strother D, Nuchtern J, Grilley B, Martingano E, Cooper K (2000) Phase I study of chemokine and cytokine gene-modified autologous neuroblastoma cells for treatment of relapsed/refractory neuroblastoma using an adenoviral vector. Hum Gene Ther 11: 1477-1488

Brodeur GM, Pritchard J, Berthold F, Carlsen NL, Castel V, Castelberry RP, De Bernardi B, Evans AE, Favrot M, Hedborg F. (1993) Revisions of the international criteria for neuroblastoma diagnosis, staging, and response to treatment. J Clin Oncol 11: 1466-1477

Coll JL, Wagner E, Combaret V, Metchler K, Amstutz H, Iacono-Di-Cacito I, Simon N, Favrot MC (1997) In vitro targeting and specific transfection of human neuroblastoma cells by chCE7 antibody-mediated gene transfer. Gene Ther 4: 156-161

Corrias MV, Basso S, Meazza R, Musiani P, Santi L, Bocca P, Occhino M, Ferrini S, Pistoia V (1998) Characterization and tumorigenicity of human neuroblastoma cells transfected with the IL-2 gene. Cancer Gene Ther 5: $38-44$

Corrias MV, Occhino M, Croce M, De Ambrosis A, Pistillo MP, Bocca P, Pistoia V, Ferrini S (2001) Lack of HLA-class I antigens in human neuroblastoma cells: analysis of its relationship to TAP and tapasin expression. Tissue Antigens 57: 110-117

Corrias MV, Scaruffi P., Occhino M, De Bernardi B, Tonini GP, Pistoia V (1996) Expression of MAGE-1, MAGE-3 and MART-1 genes in neuroblastoma. Int J Cancer 69: 403-407

Coze C, Aalto-Setala K, Brenner M, Chiang Y, Gangavalli R, Leimig T (1995) Characteristics and immunomodulatory properties of human neuroblastoma cells after retrovirus-mediated gene transfer of the cytokine genes IL-2 and IFN-g. Transgenics 1: 585-595

Forni G, Boggio K (1999) Cytokine gene-engineered vaccines. Curr Opin Mol Ther 1: 34-38

Fulda S, Debatin KM (2002) IFNgamma sensitizes for apoptosis by upregulating caspase-8 expression through the Stat1 pathway. Oncogene 21: $2295-2308$

Fulda S, Kufer MU, Meyer E, van Valen F, Dockhorn-Dworniczak B, Debatin KM (2001) Sensitization for death receptor- or drug-induced apoptosis by re-expression of caspase- 8 through demethylation or gene transfer. Oncogene 20: 5865-5877

Grossmann ME, Brown MP, Brenner M.K (1997) Antitumor responses induced by transgenic expression of CD40 ligand. Hum Gene Ther 8: $1935-1943$

\section{ACKNOWLEDGEMENTS}

This work was supported by Progetti di Ricerca Corrente and Progetto di Ricerca Finalizzata of Ministero della Salute to Gaslini Institute and Istituto Nazionale per la Ricerca sul Cancro. MC is recipient of Fondazione Italiana per la Lotta al Neuroblastoma fellowship. The excellent secretarial assistance of Ms C Bernardini and Ms A Cesarini are deeply acknowledged.

Heuer JG, Tucker-McClung C, Gonin R, Hock RA (1996) Retrovirusmediated gene transfer of B7-1 and MHC class II converts a poorly immunogenic neuroblastoma into a highly immunogenic one. Hum Gene Ther 7: 2059-2068

Hock RA, Reynolds BD, Tucker-McClung CL, Heuer JG (1996) Murine neuroblastoma vaccines produced by retroviral transfer of MHC class II genes. Cancer Gene Ther 3: 314-320

Hock RA, Reynolds BD, Tucker-McClung CL, Kwok WW (1995) Human class II major histocompatibility complex gene transfer into murine neuroblastoma leads to loss of tumorigenicity, immunity against subsequent tumor challenge, and elimination of microscopic preestablished tumors. J Immunother Emphasis Tumor Immunol 17: $12-18$

Hsieh CL, Chen DS, Hwang LH (2000) Tumor-induced immunosuppression: a barrier to immunotherapy of large tumors by cytokine-secreting tumor vaccine. Hum Gene Ther 11: 681-692

Katsanis E, Bausero MA, Panoskaltsis-Mortari A, Dancisak BB, Xu Z, Orchard PJ, Davis CG, Blazar BR (1996) Irradiation of singly and doubly transduced murine neuroblastoma cells expressing B7-1 and producing interferon-gamma reduces their capacity to induce systemic immunity. Cancer Gene Ther 3: 75-82

Katsanis E, Orchard PJ, Bausero MA, Gorden KB, McIvor RS, Blazar BR (1994) Interleukin-2 gene transfer into murine neuroblastoma decreases tumorigenicity and enhances systemic immunity causing regression of preestablished retroperitoneal tumors. J Immunother Emphasis Tumor Immunol 15: 81 - 90

Katsanis E, Xu Z, Bausero MA, Dancisak BB, Gorden KB, Davis G, Gray GS, Orchard PJ, Blazar BR (1995) B7-1 expression decreases tumorigenicity and induces partial systemic immunity to murine neuroblastoma deficient in major histocompatibility complex and costimulatory molecules. Cancer Gene Ther 2: 39-46

Lamant L, Pulford K, Bischof D, Morris SW, Mason DY, Delsol G, Mariame B (2000) Expression of the ALK tyrosine kinase gene in neuroblastoma. Am J Pathol 156: $1711-1721$

Lampson LA, Fisher CA, Whelan JP (1983) Striking paucity of HLA-A, B, C and beta 2 -microglobulin on human neuroblastoma cell lines. J Immunol 130: $2471-2478$

Lode HN, Pertl U, Xiang R, Gaedicke G, Reisfeld RA (2000) Tyrosine hydroxylase-based DNA-vaccination is effective against murine neuroblastoma. Med Pediatr Oncol 35: 641-646

Loh JE, Chang CH, Fodor WL, Flavell RA (1992) Dissection of the interferon gamma-MHC class II signal transduction pathway reveals that type I and type II interferon systems share common signalling component(s). EMBO J 11: 1351-1363

Montaldo PG, Carbone R, Corrias MV, Ferraris PC, Ponzoni M (1994) Synergistic differentiation-promoting activity of interferon gamma and tumor necrosis factor-alpha: role of receptor regulation on human neuroblasts. J Natl Cancer Inst 86: 1694-1701

Nitta T, Ebato M, Sato K, Okumura K (1994) Expression of tumour necrosis factor-alpha, -beta and interferon-gamma genes within human neuroglial tumour cells and brain specimens. Cytokine 6: 171-180

Pardoll DM (2000) Therapeutic vaccination for cancer. Clin Immunol 95: S44-62

Parmiani G, Rodolfo M, Melani C (2000) Immunological gene therapy with ex vivo gene-modified tumor cells: a critique and a reappraisal. Hum Gene Ther 11: $1269-1275$

Ponzoni M, Casalaro A, Lanciotti M, Montaldo PG, Cornaglia-Ferraris M (1992) The combination of gamma-interferon and tumor necrosis factor causes a rapid and extensive differentiation of human neuroblastoma cells. Cancer Res 52: 931 - 939

Ponzoni M, Guarnaccia F, Corrias MV, Cornaglia-Ferraris P (1993) Uncoordinate induction and differential regulation of HLA class-I and 
class-II expression by gamma-interferon in differentiating human neuroblastoma cells. Int J Cancer 55: 817-823

Refaeli Y, Van Parijs L, Alexander SI, Abbas AK (2002) Interferon gamma is required for activation-induced death of T lymphocytes. J Exp Med 196: 999- 1005

Rimoldi D, Romero P, Carrel S (1993) The human melanoma antigenencoding gene, MAGE-1, is expressed by other tumour cells of neuroectodermal origin such as glioblastomas and neuroblastomas. Int J Cancer 54: $527-528$

Rodolfo M, Luksch R, Stockert E, Chen YT, Collini P, Ranzani T, Lombardo C, Dalerba P, Rivoltini L, Arienti F, Fossati-Bellani F, Old LJ, Parmiani G, Castelli C (2003) Antigen-specific immunity in neuroblastoma patients: antibody and T-cell recognition of NY-ESO-1 tumor antigen. Cancer Res 63: $6948-6955$

Rosenthal FM, Cronin K, Bannerji R, Golde DW, Gansbacher B (1994) Augmentation of antitumor immunity by tumor cells transduced with a retroviral vector carrying the interleukin-2 and interferon-gamma cDNAs. Blood 83: 1289-1298

Rousseau RF, Haight AE, Hirschmann-Jax C, Yvon ES, Rill DR, Mei Z Smith SC, Inman S, Cooper K, Alcoser P, Grilley B, Gee A, Popek E, Davidoff A, Bowman LC, Brenner MK, Strother D (2003) Local and systemic effects of an allogeneic tumor cell vaccine combining transgenic human lymphotactin with interleukin-2 in patients with advanced or refractory neuroblastoma. Blood 101: 1718-1726

Sarkar AK, Burlingame SM, Zang YQ, Dulai V, Hicks MJ, Strother DR Nuchtern JG (2001) Major histocompatibility complex-restricted lysis of neuroblastoma cells by autologous cytotoxic T lymphocytes. J Immunother 24: $305-311$
Sgadari C, Angiolillo AL, Tosato G (1996) Inhibiton of angiogenesis by interleukin-12 is mediated by the interferon-inducible protein 10 . Blood 87: $3877-3882$

Soling A, Schurr P, Berthold F (1999) Expression and clinical relevance of NY-ESO-1, MAGE-1 and MAGE-3 in neuroblastoma. Anticancer Res 19 $2205-2209$

Thiele C (1999) Neuroblastoma, Vol. 1. Human Cell Culture London: Kluwer Academic Publishers

Traversari C, Meazza R, Coppolecchia M, Basso S, Verrecchia A, van der Bruggen P, Ardizzoni A, Gaggero A, Ferrini S (1997) IFN-gamma gene transfer restores HLA-class I expression and MAGE-3 antigen presentation to CTL in HLA-deficient small cell lung cancer. Gene Ther 4: $1029-1035$

Ucar K, Seeger RC, Challita PM, Watanabe CT, Yen TL, Morgan JP, Amado R, Chou E, McCallister T, Barber JR, Jolly DJ, Reynolds CP, Gangavalli R, Rosenblatt JD (1995) Sustained cytokine production and immunophenotypic changes in human neuroblastoma cell lines transduced with a human gamma interferon vector. Cancer Gene Ther 2: 171-181

Wadhwa PD, Zielske SP, Roth JC, Ballas CB, Bowman JE, Gerson S.L (2002) Cancer gene therapy: scientific basis. Annu Rev Med 53: 437-452

Watanabe Y, Kuribayashi K, Miyatake S, Nishihara K, Nakayama E, Taniyama T, Sakata T (1989) Exogenous expression of mouse interferon gamma cDNA in mouse neuroblastoma C1300 cells results in reduced tumorigenicity by augmented anti-tumor immunity. Proc Natl Acad Sci USA 86: $9456-9460$

Yang KD, Shaio MF, Wang CL, Wu NC, Stone RM (1994) Neuroblastoma cell-mediated leukocyte chemotaxis: lineage-specific differentiation of interleukin-8 expression. Exp Cell Res 211: 1 -5 